\title{
Enhanced thermal performance of garments embedded with encapsulated phase change material
}

\author{
John A. Gear* Michael J. Lachut ${ }^{\dagger} \quad$ Yan Ding* $^{*}$
}

(Received 17 November 2005; revised 20 July 2006)

\begin{abstract}
The thermal storage and insulation properties of garments enhanced with phase change material (PCM) will be investigated using a finite difference procedure. A diver dry suit embedded with microencapsulated PCM will be shown to enhance thermal protection under extreme temperature conditions. Under conditions of high body heat production a garment embedded with Macro-encapsulated PCM is shown to absorb excess heat while maintaining a relatively constant temperature.
\end{abstract}

*School of Mathematical and Geospatial Sciences, RMIT University, Melbourne, Australia. mailto: jag@rmit.edu.au

$\dagger$ Dept. Mathematics and Statistics, University of Melbourne, Melbourne, Australia.

See http://anziamj.austms.org.au/V47EMAC2005/Gear for this article, (C) Austral. Mathematical Soc. 2006. Published July 29, 2006. ISSN 1446-8735 


\section{Contents}

1 Introduction

C138

2 Governing equations

C139

3 Finite difference scheme

C142

4 Results

C144

4.1 Diver dry suit . . . . . . . . . . . . . .

C144

4.2 Cooling garment

5 Conclusion

C150

References

C150

\section{Introduction}

When Phase Change Materials (PCMs) are added to foams or fabrics, new materials are created that provide both enhanced thermal storage and insulation $[2,4,5,6]$. They add thermal capacitance by taking advantage of the latent heat release (or absorption) when the PCM changes phase.

Applications for garments enhanced with PCMs may include hiker clothing, ski clothing, athletic clothing and diver dry suits. In each case the aim would be to keep the wearer warmer or cooler, or even both, over an extended period [2].

Due to their large surface area (of the order of square meters) and their small thickness (between a few millimeters and a centimeter), garments embedded with PCMs can be modelled as one-dimensional heat flow in a continuum. Here we investigate the heat flow using a one-dimensional finite difference procedure. The thermal protection of a diver dry suit constructed 
from a foam enhanced with micro-encapsulated PCM and standard insulation will be investigated [6]. Layering of PCM foam and standard insulation will be compared. In conditions of high heat production garments embedded with macro-encapsulated PCM are shown to absorb excess heat while maintaining a relatively constant temperature.

\section{Governing equations}

Let $u=u(x, t)$ be the temperature $\left[{ }^{\circ} \mathrm{K}\right]$ and $h=h(u)$ the enthalpy per unit volume $\left[\mathrm{J} \cdot \mathrm{m}^{-3}\right]$. If $k=k(u)$ is the thermal conductivity $\left[\mathrm{W} \cdot \mathrm{m}^{-1} \cdot{ }^{\circ} \mathrm{K}^{-1}\right]$, then by Fourier's law of heat conduction the rate of flow of heat or flux of heat $\left[\mathrm{W} . \mathrm{m}^{-2}\right]$ in one dimension is proportional to the gradient of the temperature,

$$
q=-k(u) \frac{\partial u}{\partial x} .
$$

The one-dimensional heat equation is [3, p.304]

$$
\frac{\partial h}{\partial t}=\frac{\partial}{\partial x}\left(k(u) \frac{\partial u}{\partial x}\right) .
$$

The enthalpy $h(u)$ is essentially the heat content per unit volume. Defining the enthalpy relative to $0^{\circ} \mathrm{C}$, then $h(u)$ represents the quantity of heat required to raise a unit volume from 0 to $u^{\circ} \mathrm{C}$. For heat conduction, with no phase changes or moving boundaries, the enthalpy is $h(u)=\rho \int_{0}^{u} c\left(u^{\prime}\right) d u^{\prime}$ where $\rho$ is the constant density and $c(u)$ is the specific heat $\left[\mathrm{J}^{\mathrm{kgg}}{ }^{-1} \cdot{ }^{\circ} \mathrm{K}^{-1}\right]$. Note that for constant specific heat $(h=\rho c u)$ and constant thermal conductivity, Equation (1) reduces to the usual one-dimensional heat equation

$$
\frac{\partial u}{\partial t}=\kappa \frac{\partial^{2} u}{\partial x^{2}},
$$

where $\kappa=k /(\rho c)$ is the thermometric conductivity. 
For phase change problems the situation is slightly more complicated because of latent heat. For simplicity we assume that the thermal conductivity $k(u)$ is constant in each phase, that is

$$
k(u)= \begin{cases}k_{s}, & u<u_{f}, \\ k_{l}, & u>u_{f} .\end{cases}
$$

Here the fusion temperature is $u_{f}$, and $k_{s}$ and $k_{l}$ are constant thermal conductivities in the solid and liquid phases respectively. If we also assume constant specific heat $c(u)$ in each phase and apply a constant heat flux condition at the phase change interface, which can be determined using a Stefan condition (energy balance) [3], then we could use the heat equation (2) to determine the temperature profile for all time.

The enthalpy, temperature and the flux of heat are all continuous across the phase change interface (the temperature gradient is discontinuous). Hence we determine the temperature profile for all time using Equation (1) without needing any explicit reference to the position of the phase change interface. Unfortunately the partial differential equation is slightly more complicated and the finite difference scheme must be explicit, which restricts the size of the time step (see $\S 3$ ).

For the PCMs considered in $\S 4$, the phase change occurs over a temperature range of about $1^{\circ} \mathrm{K}$ and the thermal conductivities and specific heats vary only slightly with temperature over the range of temperatures considered here $\left(4-36^{\circ} \mathrm{C}\right)$. The most significant feature which determines the thermal protection, or temperature control properties of the garment is the latent heat $L\left[\mathrm{~J}_{\mathrm{kg}}-1\right]$ (the jump in enthalpy at the phase change is $\rho L$ ). Let $c_{s}$ and $c_{l}$ be constant specific heats in the solid and liquid phases, respectively, the enthalpy [3]

$$
h(u)= \begin{cases}\rho c_{s} u, & u<u_{f}, \\ {\left[\rho c_{s} u_{f}, \rho\left(c_{s} u_{f}+L\right)\right],} & u=u_{f}, \\ \rho\left(L+\left(c_{s}-c_{l}\right) u_{f}\right)+\rho c_{l} u, & u>u_{f},\end{cases}
$$




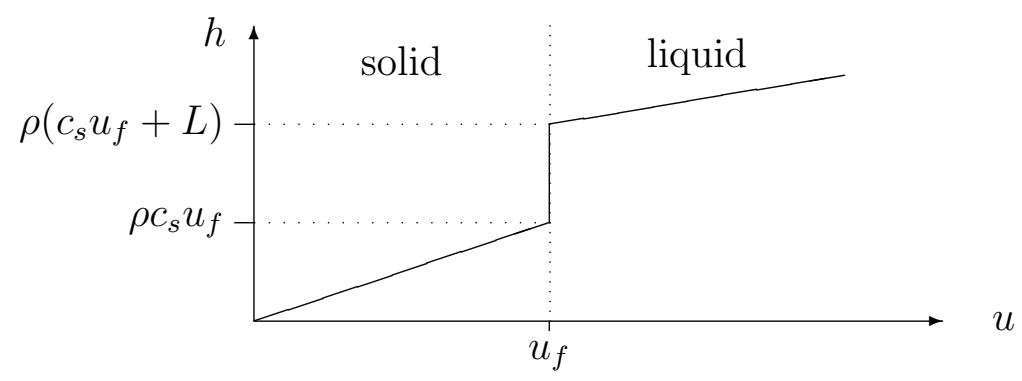

FiguRE 1: Enthalpy versus temperature.

where $[a, b]$ denotes that $h(u)$ has any value in the interval $a \leq h(u) \leq b$, see Figure 1.

In a strict sense the enthalpy is not a function of temperature, see Figure 1, but, for any value of $h$ there corresponds one and only one temperature $u$, so that temperature is a function of enthalpy. So given the temperature profile at any time $t$ we step forward in time to $t+\Delta t$ using Equation (1) and determine the enthalpy $h(u)$. The temperature profile at $t+\Delta t$ is then uniquely determined from Equation (3).

We assume Newton cooling at the air boundary $x=0$, then

$$
k(u) \frac{\partial u}{\partial x}(0, t)=P\left[u(0, t)-u_{A}\right]
$$

where $P\left[\mathrm{~W} . \mathrm{m}^{-2} \cdot{ }^{\circ} \mathrm{K}^{-1}\right]$ is the surface heat transfer coefficient or surface conductance and $u_{A}$ is the ambient temperature.

At the skin boundary $x=w$, we have two possible simple strategies. In an extreme temperature environment we are interested in determining the rate of heat loss. For example, how long can an Arctic construction worker stay working outside in extreme cold. In this situation it is reasonable to assume constant skin temperature over an extended time period and calculate the flux of heat at the skin boundary.

In a situation of high heat production, like an athlete resting during competition, we are interested in determining the cooling effect of a garment. 
For a short time period it would be reasonable to assume constant heat flux at the skin boundary. During this period we would calculate the change in skin temperature.

\section{Finite difference scheme}

The region $0 \leq x \leq w$ is divided into $N$ intervals of width $\Delta x=w / N$ and we define that $u_{i}^{j}$ is an average temperature on $\left(i-\frac{1}{2}\right) \Delta x \leq x \leq\left(i+\frac{1}{2}\right) \Delta x$, $i=1,2, \ldots, N$, at time $t=j \Delta t$. Using a first order forward difference in time and conservative second order spatial differences Equation (1) is discretized as

$$
\tilde{h}_{i}^{j+1}=\tilde{h}_{i}^{j}+\frac{\Delta t}{\rho c_{s}(\Delta x)^{2}}\left[k_{i+\frac{1}{2}}\left(u_{i+1}^{j}-u_{i}^{j}\right)-k_{i-\frac{1}{2}}\left(u_{i}^{j}-u_{i-1}^{j}\right)\right],
$$

where $\tilde{h}=h /\left(\rho c_{s}\right)$ (a scaled form of the enthalpy) and

$$
k_{i+\frac{1}{2}}= \begin{cases}k_{s}, & \text { if }\left(u_{i+1}^{j}+u_{i}^{j}\right)<2 u_{f}, \\ k_{l}, & \text { if }\left(u_{i+1}^{j}+u_{i}^{j}\right) \geq 2 u_{f} .\end{cases}
$$

Given the temperature profile $u_{i}^{j}$ and scaled enthalpy $\tilde{h}_{i}^{j}$ for all $i$ at time $j \Delta t$, Equation (5) with (6) determines the scaled enthalpy $\tilde{h}_{i}^{j+1}$ for all $i$ at time $(j+1) \Delta t$. The temperature profile at time $(j+1) \Delta t$ is then

$$
u_{i}^{j+1}= \begin{cases}\tilde{h}_{i}^{j+1}, & \text { if } \tilde{h}_{i}^{j+1}<u_{f}, \\ u_{f}, & \text { if } u_{f} \leq \tilde{h}_{i}^{j+1}<u_{f}+L / c_{s}, \\ u_{f}+\frac{c_{s}}{c_{l}}\left(\tilde{h}_{i}^{j+1}-u_{f}-L / c_{s}\right), & \text { if } \tilde{h}_{i}^{j+1} \geq u_{f}+L / c_{s} .\end{cases}
$$

For heat conduction without phase change the scheme (5) is easily shown to be numerically stable when $\frac{\Delta t}{(\Delta x)^{2}} \kappa<\frac{1}{2}, \kappa=k / \rho c[1, \S 2.1]$. Numerical 
experiment on (5) with (6) and (7), confirm the same stability boundary for heat conduction with phase change, but now $\kappa=\max \left\{k_{s} / \rho c_{s}, k_{l} / \rho c_{l}\right\}$.

Let the air boundary $x=0$ lie half way between node points $i=0$ and 1 . The node $i=0$ will be a fictitious level outside the body. Using a central difference for the first order derivative in Equation (4) and an average over nodes 0 and 1 for $u(0, t)$ we obtain

$$
u_{0}=-u_{1}+2 u_{A}+2 \frac{u_{1}-u_{A}}{1+\frac{P \Delta x}{2 k}},
$$

where the superscript $j$ is omitted for clarity. Note that if $P \rightarrow 0$, then $u_{0} \rightarrow u_{1}$ and as expected there is no flux of heat across the boundary. If $P \rightarrow \infty$, then $\frac{1}{2}\left(u_{0}+u_{1}\right) \rightarrow u_{A}$ or equivalently $u(0, t) \rightarrow u_{A}$, again as expected.

If a fixed temperature condition is used at the skin boundary $x=w$, then we set the boundary half way between node points $i=N$ and $N+1$. Again, node $i=N+1$ is fictitious and only necessary so that the boundary condition can be satisfied. Then

$$
u_{N+1}=-u_{N}+2 u_{S}
$$

where $u_{S}$ is the fixed temperature at the skin boundary. The heat flux at the boundary is calculated using $k\left(u_{S}-u_{N}\right) /(\Delta x / 2)$. If alternatively heat flux at the skin boundary is constant and given by $F$, then

$$
u_{N+1}=-u_{N}+F \Delta x / k,
$$

and $u_{N}$ is the skin boundary temperature.

At a boundary between layers (in a composite fabric/foam) there must be continuous temperature and heat flux. Assuming a boundary between layers lies half way between nodes $i=i_{s}$ and $i_{s}+1$, then

$$
\tilde{u}_{i_{s}+1}+u_{i_{s}}=u_{i_{s}+1}+\tilde{u}_{i_{s}}, \quad k^{-}\left(\tilde{u}_{i_{s}+1}-u_{i_{s}}\right)=k^{+}\left(u_{i_{s}+1}-\tilde{u}_{i_{s}}\right) .
$$




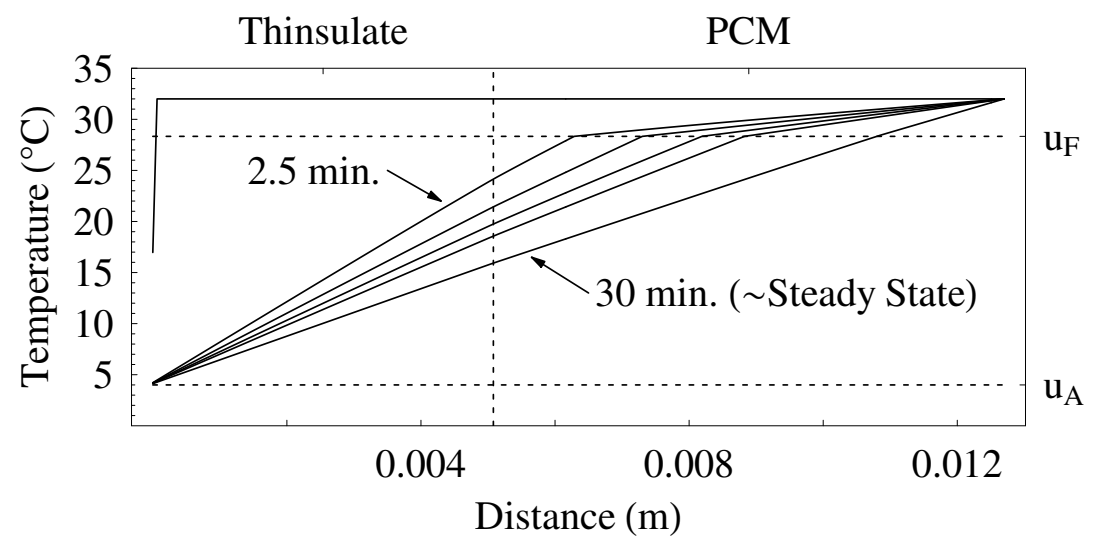

Figure 2: Temperature profile, 2.5 minute intervals for 10 minutes and at 30 minutes. Outer $40 \%$ Thinsulate $+60 \%$ PCM

These equations are solved for the fictitious values $\tilde{u}_{i_{s}}$ and $\tilde{u}_{i_{s}+1}$. Then, when $i=i_{s}$, the finite difference procedure is modified so that $u_{i_{s}+1}=\tilde{u}_{i_{s}+1}$ and similarly when $i=i_{s}+1, u_{i_{s}}=\tilde{u}_{i_{s}}$.

\section{Results}

\subsection{Diver dry suit}

Consider a diver dry suit constructed from a foam enhanced with microencapsulated phase change material C18 paraffin (octadecane) in Thermosorb ${ }^{1}$ and a micro-fibrous insulating material called Thinsulate. ${ }^{2}$ The suit has thickness $0.0127 \mathrm{~m}$. The initial temperature of the suit is $32^{\circ} \mathrm{C}$. The divers skin temperature is considered to be constant at $32^{\circ} \mathrm{C}$. The diver and suit

${ }^{1}$ Thermosorb is a trade name used by Frisby Technologies of Clemmons, NC.

${ }^{2}$ Thinsulate is a trademark of the $3 \mathrm{M}$ Corporation 


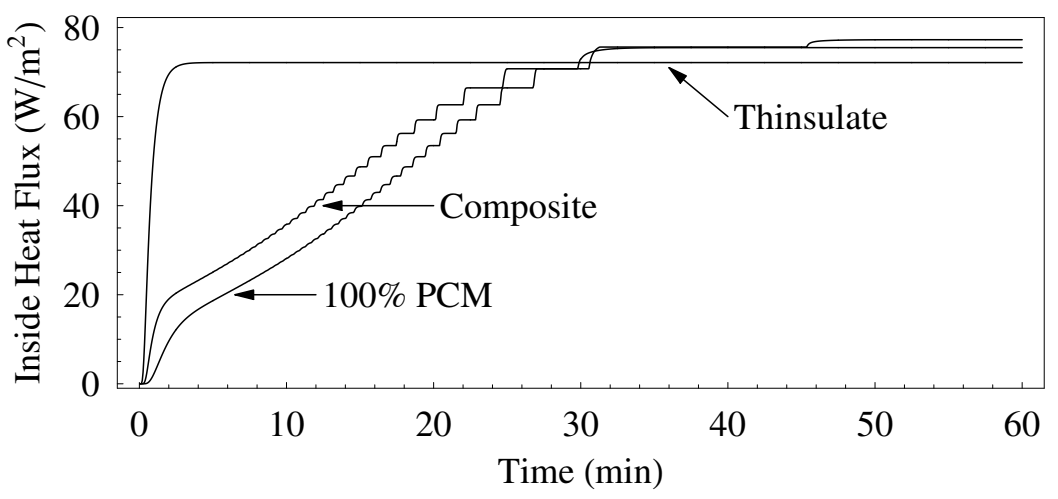

Figure 3: Inside heat flux against time for 100\% Thinsulate, $40 \%$ Thinsulate $+60 \%$ PCM (Composite) and 100\% PCM.

are immersed in water of $4^{\circ} \mathrm{C}$. Typical values of surface heat transfer coefficient, for icy water, range from 100 to $1000\left[\mathrm{~W} \cdot \mathrm{m}^{-2} .{ }^{\circ} \mathrm{K}^{-1}\right]$. In the following numerical experiments we use $P=600\left[\mathrm{~W} \cdot \mathrm{m}^{-2} \cdot{ }^{\circ} \mathrm{K}^{-1}\right]$. Thermal conductivities, specific heats, densities, latent heat and fusion temperature are given in Table 1.

We initially consider the same example used by Nuckols [6]. The suits outer layer is Thinsulate (40\%) and inner layer is PCM foam. The phase change material is initially all liquid. A grid of 100 points was used. In Figure 2 the temperature profiles are plotted for 2.5 minute intervals for the first 10 minutes and at 30 minutes. The profile at 30 minutes is very close to the steady state solution. Notice that after 2.5 minutes the temperature in the Thinsulate layer has significantly dropped and that the inner layer temperature is being maintained by the latent heat being released as the PCM changes from liquid to solid.

Figure 3 shows a comparison of the inside heat flux against time, between the composite material ( $40 \%$ Thinsulate $+60 \%$ PCM), 100\% Thinsulate and $100 \%$ PCM. The results for the composite and $100 \%$ Thinsulate are similar 


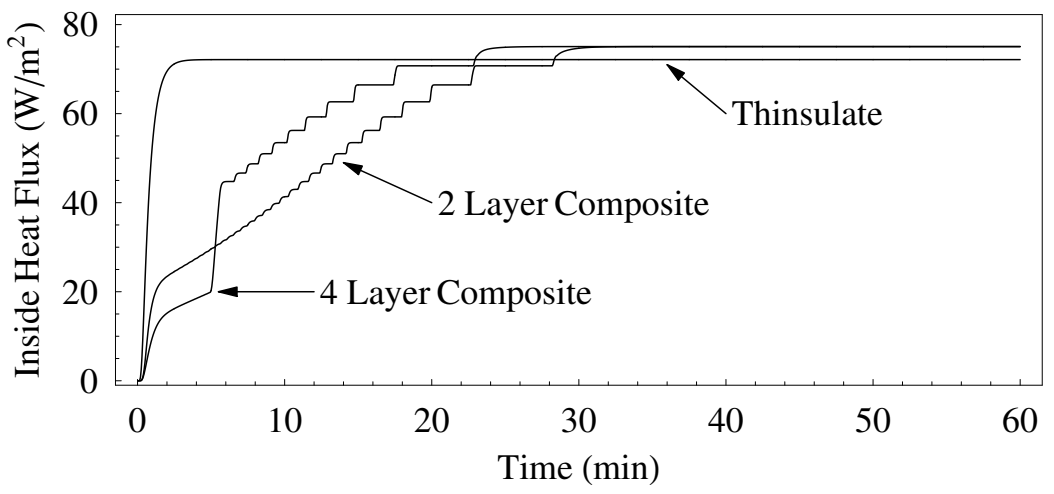

Figure 4: Inside heat flux against time for Thinsulate only, two layer Thinsulate/PCM composite and four layer Thinsulate/PCM composite.

\begin{tabular}{|c|c|c|c|}
\hline & PCM solid & PCM liquid & Thinsulate \\
\hline \multirow{3}{*}{$\begin{array}{l}\text { Specific heat }\left[\mathrm{J} \mathrm{kg}^{-1} \cdot{ }^{\circ} \mathrm{K}^{-1}\right] \\
\text { Therm. conductivity }\left[\mathrm{W} \cdot \mathrm{m}^{-1} \cdot{ }^{\circ} \mathrm{K}^{-1}\right] \\
\text { Density }\left[\mathrm{kg} \cdot \mathrm{m}^{-3}\right]\end{array}$} & 1483 & 1620 & \multirow{5}{*}{$\begin{array}{c}1129.68 \\
0.03286 \\
52.86\end{array}$} \\
\hline & 0.03478 & 0.03798 & \\
\hline & \multirow{3}{*}{\multicolumn{2}{|c|}{$\begin{array}{c}121.43 \\
99424.87 \\
28.3333\end{array}$}} & \\
\hline Latent heat $\left[{\left.\mathrm{J} . \mathrm{kg}^{-1}\right]}^{-1}\right.$ & & & \\
\hline Fusion Temp. ${ }^{\circ} \mathrm{C}$ & & & \\
\hline \multicolumn{2}{|l|}{$u_{A}=4^{\circ} \mathrm{C}$} & \multicolumn{2}{|c|}{$w=0.0127 \mathrm{~m}$} \\
\hline
\end{tabular}

\begin{tabular}{|c|l|l|l|}
\hline & Grid & $\Delta t[\mathrm{sec}]$ & $\Delta x[\mathrm{~m}]$ \\
\hline 100\% Thinsulate & 50 & .05 & .000254 \\
$40 \%$ Thinsulate $60 \% \mathrm{PCM}$ & $40+60$ & .0125 & .000127 \\
$50 \%$ Thinsulate $50 \% \mathrm{PCM}$ & $50+50$ & .0125 & .000127 \\
4 Layer Composite & $25+25+25+25$ & .0125 & .000127 \\
$100 \%$ PCM & 100 & .0375 & .000127 \\
\hline
\end{tabular}

TABLE 1: Thermal properties and numerical constants for the diver dry suit results. 


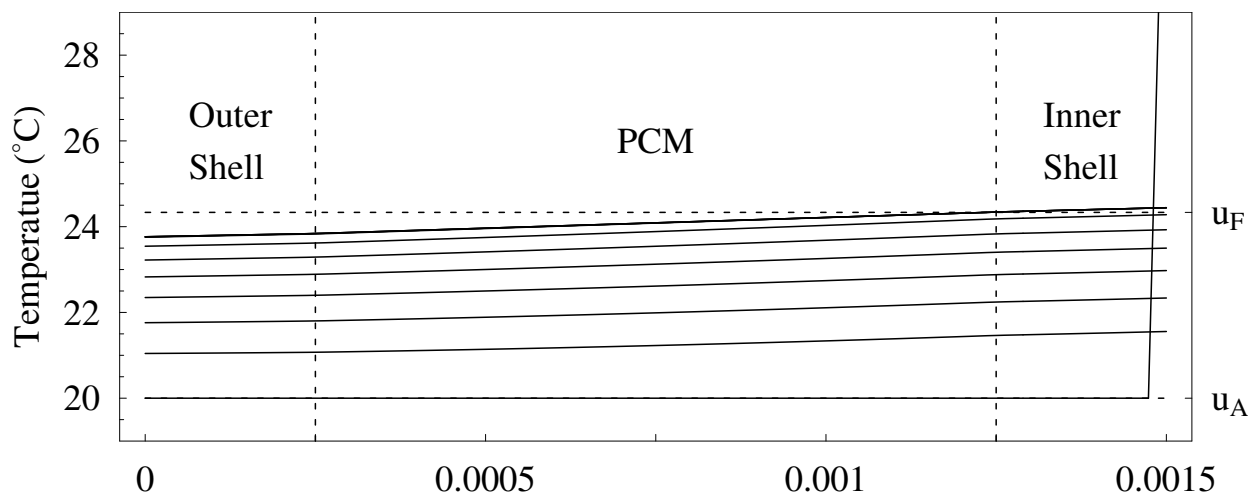

Distance (m)

Figure 5: Temperature against distance every 0.5 minutes for 5 minutes. Initial temperature and air temperature are $20^{\circ} \mathrm{C}$. Phase change temperature is $24 \frac{1}{3}^{\circ} \mathrm{C}$.

to those in [6]. Notice that within about 3 minutes the temperature gradient at the skin boundary for the $100 \%$ Thinsulate has reached its steady state value. Hence a diver wearing a dry suit of Thinsulate will feel the effects of the cold water within 3 minutes. Both the composite and 100\% PCM protect the diver for much longer, up to 30 minutes. The stepping in the composite and $100 \%$ PCM plots is due to the grid spacing and the movement of the phase change interface between node points in the grid. Grids of 50, 100 and 200 points where investigated with no appreciable difference in results, apart from the step distance halving each time the grid doubled.

Colvin \& Bryant [2] presented experimental results for multiple layered composite garments, we expect the layering will enhance thermal protection. In Figure 4 we compare the inside heat flux against time for $100 \%$ Thinsulate, a composite of $50 \%$ Thinsulate (outer layer) and $50 \% \mathrm{PCM}$, and a four layer composite of Thinsulate and PCM. Initially the four layer composite offers greater thermal protection as it has a thinner outer layer of Thinsulate and hence the first layer of PCM releases its latent heat earlier. But, after about 


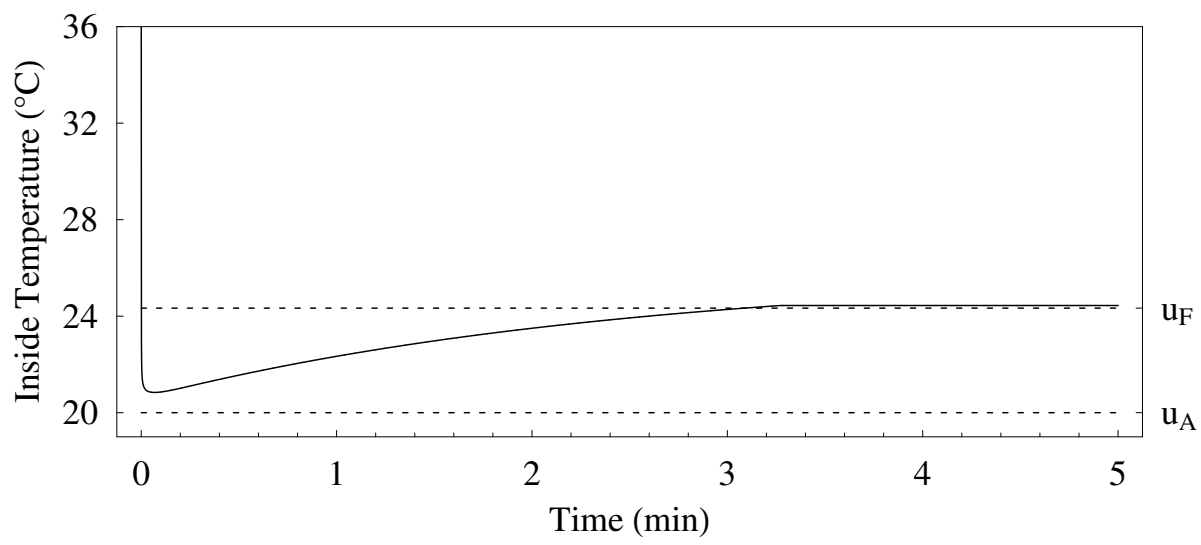

FigURE 6: Inside temperature against time. Initial temperature and air temperature are $20^{\circ} \mathrm{C}$. Phase change temperature is $24 \frac{1}{3}^{\circ} \mathrm{C}$.

6 minutes the two layer composite has significantly greater protection.

\subsection{Cooling garment}

Macro-encapsulated phase change material are proposed for use in garments designed for cooling [2]. The phase change temperature can be adjusted by blending various paraffins [4]. For example, hexadecane and octadecane have fusion temperatures of $18 \frac{1}{3}^{\circ} \mathrm{C}$ and $28 \frac{1}{3}^{\circ} \mathrm{C}$ respectively [6].

Consider a cooling garment constructed of outer shell $0.25 \mathrm{~mm}, 1 \mathrm{~mm}$ PCM and inner shell of $0.25 \mathrm{~mm}$, See Table 2 for the thermal properties. We assume that the individual is hot and active with initial skin temperature of $36^{\circ} \mathrm{C}$, constant heat flux of 100 Watts $/ \mathrm{m}^{2}$ (180 Watts over an area of $\left.1.8 \mathrm{~m}^{2}\right)$ and air temperature is $20^{\circ} \mathrm{C}$. Figures 5 and 6 show results when the cooling garment is initially at air temperature $20^{\circ} \mathrm{C}$ and the fusion temperature is set at $24 \frac{1}{3}^{\circ} \mathrm{C}$. Figure 5 shows the temperature against distance every 30 seconds for the first five minutes. The lowest line is the first temperature profile. 


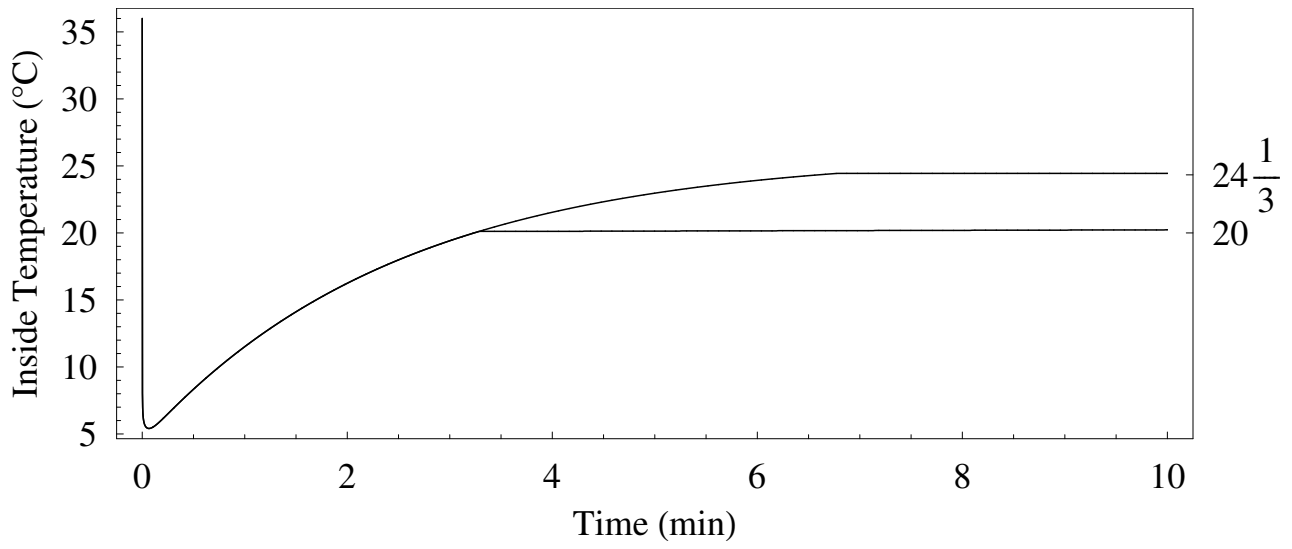

Figure 7: Inside temperature against time. Initial temperature $4^{\circ} \mathrm{C}$. Air temperature $20^{\circ} \mathrm{C}$. Phase change temperatures $24 \frac{1}{3}^{\circ} \mathrm{C}$ and $20^{\circ} \mathrm{C}$

\begin{tabular}{|c|c|c|c|}
\hline & PCM solid & PCM liquid & shell \\
\hline Specific heat $\left[\mathrm{J} \cdot \mathrm{kg}^{-1} \cdot{ }^{\circ} \mathrm{K}^{-1}\right]$ & 1882 & 2213 & 1673 \\
\hline Therm. conductivity $\left[\mathrm{W} \cdot \mathrm{m}^{-1} \cdot{ }^{\circ} \mathrm{K}^{-1}\right]$ & 0.1505 & 0.1505 & 0.25 \\
\hline Density $\left[\mathrm{kg} \cdot \mathrm{m}^{-3}\right]$ & 903.5 & 773.8 & 1330 \\
\hline Latent heat $\left[\mathrm{J} \mathrm{kg}^{-1}\right]$ & \multicolumn{2}{|c|}{239418} & \\
\hline
\end{tabular}

\begin{tabular}{|l|c|c||l|l|c|}
\hline & & Grid Points & & & Grid Points \\
\hline Outer shell & $0.25 \mathrm{~mm}$ & 10 & PCM & $1 \mathrm{~mm}$ & 38 \\
Inner shell & $0.25 \mathrm{~mm}$ & 10 & Width $w$ & $1.5 \mathrm{~mm}$ & 58 \\
\hline
\end{tabular}

TABLE 2: Thermal properties and numerical constants for the cooling suit results. 
As time increases the temperature in the garment increases. The last four lines coincide so the temperature profile is constant after about 3.5 minutes. Figure 6 shows the inside temperature against time. We initially see a large drop in temperature. But again after about 3 minutes the inside temperature remains constant at the fusion temperature.

In Figure 7 we show inside temperature against time for garments with initial temperature of $4^{\circ} \mathrm{C}$ and fusion temperatures of $24 \frac{1}{3}^{\circ} \mathrm{C}$ and $20^{\circ} \mathrm{C}$. The initial drop in inside temperature is now much larger. But the final temperatures are still controlled by the fusion temperatures. The results show that initial temperature of the garment is not important (provided that it is less than the fusion temperature) and that we can control the inside temperature by carefully selecting the fusion temperature of the PCM.

\section{Conclusion}

Micro-encapsulated phase change material embedded in a diver dry suit enhances thermal protection when compared with Thinsulate and should enable divers to work for longer in extreme temperature conditions. Layering of garments does not appear to enhance thermal protection.

Macro-encapsulated phase change material in a cooling garment can be used (to some extent) to control body temperature. The inside temperature of the garment can be controlled by carefully selecting the fusion temperature of the PCM.

\section{References}

[1] W. F. Ames. Numerical Methods for Partial Differential Equations. Academic Press, second edition, 1977. C142 
[2] D. P. Colvin and Y. G. Bryant. Protective clothing containing encapsulated phase change materials. Advances in Heat and Mass Transfer in Biotechnology, HD-Vol.362/BED-Vol. 40:123-132, 1998. C138, C147, C148

[3] J. M. Hill and J. N. Dewynne. Heat Conduction. Applied mathematics and engineering science texts. Blackwell Scientific Publications, 1987. C139, C140

[4] D. C. Hittle and T. L. André. A new test instrument and procedure for evaluation of fabrics containing phase-change material. ASHRAE Transactions, 4509:175-182, 2002. C138, C148

[5] G. E. R. Lamb and K. Duffy-Morris Heat loss through fabrics under ventilation with and without a phase transistion additive. Textile Research Journal, pages 261-265, 1990. ISSN: 0040-5175. C138

[6] M. L. Nuckols. Analytical modelling of a diver dry suit enhanced with micro-encapsulated phase change materials. Ocean Engineering, 26:547-564, 1999. http://dx.doi.org/10.1016/S0029-8018(98)00001-8 C138, C139, C145, C147, C148 